\title{
Valoración Económica de los Helechos Silvestres del Parque Nacional La Tigra, Honduras, C.A.
}

\author{
Ruth Karina Hernández-Cibrián ${ }^{1}$ y Beatriz Urbano López De Meneses ${ }^{2}$ \\ 'Universidad Nacional Autónoma de Honduras (UNAH). \\ 2Departamento de Ingeniería Agrícola y Forestal. Universidad de Valladolid (UVa).
}

\section{Correspondencia}

R.K. Hernández-Cibrián

e-mail: rkhcibrian@gmail.com

Recibido: 26 julio 2017

Aceptado: 5 septiembre 2017

Publicado on-line: diciembre 2017

\section{Resumen}

El parque nacional La Tigra (PNLT), se encuentra ubicado a $12 \mathrm{~km}$ al NE de Tegucigalpa, posee una extensión de $240,4 \mathrm{~km}^{2}$ de los cuales $164,5 \mathrm{~km}^{2}$ corresponden a la zona de amortiguamiento y $75,9 \mathrm{~km}^{2}$ corresponden a la zona núcleo, es un bosque nublado que se caracteriza por tener una gran diversidad de especies, muchas de las cuales se encuentran en peligro de extinción ya sea por el avance de la agricultura migratoria, incendios forestales, extracción ilícita entre otros. Considerando que el PNLT dispone de dos investigaciones sobre la diversidad de helechos existente en el sendero La Esperanza y La Cascada (los más visitados por los turistas) y que algunas de estas especies se encuentran en el apéndice II de CITES y que son extraídas ilegalmente para ser comercializadas en algunos viveros y mercados de la ciudad, se decidió realizar una investigación utilizando el Método de Valoración contingente (MVC), mediante el cual se realizó un cuestionario de 12 preguntas orientadas a conocer la Disposición a Pagar (DAP), por un helecho del medio silvestre. Se entrevistó un total de 68 individuos, el 91,5\% vivían en la ciudad de Tegucigalpa, el 91\% conocía los helechos y el 71,6\% compraría un helecho del medio silvestre.

El precio promedio que estarían DAP estuvo entre L. 200,00 (8,08 €) a L. 350,00 $(14,16 €)$ y el máximo precio que estarían DAP es de L.479,24 (19,38 €). Con este precio de referencia se recomienda a la organización comanejadora del PNLT o a la institución administradora de los recursos naturales, el Instituto Nacional de Conservación y Desarrollo Forestal Áreas Protegidas y Vida Silvestre (ICF), para que utilice esta información, ya sea para hacer una mejor gestión de los helechos y/o realizar investigaciones orientadas a conocer el potencial económico de estos y así desarrollar un proyecto de aprovechamiento sostenible.

Palabras clave: Helechos, silvestre, valoración contingente, disponibilidad a pagar.

\footnotetext{
Abstract

Economic Assessment of the Wild Ferns of La Tigra National Park, Honduras, C.A.

La Tigra National Park, is located at 12 kilometers northeast Tegucigalpa, it has an extension of $240.4 \mathrm{~km}^{2}$ from those $164,5 \mathrm{~km}^{2}$ corresponds to the buffer zone and $75,9 \mathrm{~km}^{2}$ corresponds to the nucleus zone, it is a cloud forest that is characterized for have a big diversity of species, most of them are in endangered due to the advance or the migratory agriculture, forest fires illegal extraction among other. Considering that La Tigra National Park disposes of two investigations about the ferns diversity that exist in La Esperanza and La Cascada path (the most visited for the tourists) and that some of the species can be found in the CITES appendix II and they are illegal extracted to be commercialized in some vivariums and markets in the city it was decided to make this investigation, using the contingent valuation method by which it
} 
was made a twelve question questionnaire oriented to know the disposition payment, for a wild fern. There were 68 interviewed persons, 91, 5\% lived in the city of Tegucigalpa, $91 \%$ knew the ferns, and $71,6 \%$ would buy a wild ferns. The average price their would be able to pay its $200 \mathrm{~L}(8 €)$, to $350 \mathrm{~L}(14,16 €)$ and the maximum price they would pay its $479 \mathrm{~L}(19,38 €)$. With this price reference it is recommended to the organization that co-handler, or the institution that controls the natural resources (ICF) in Honduras to let them use this information, either to make a better ferns management, or to investigations oriented to know the economic potential of this, and in this way develop a sustainable project.

Key words: Ferns, wild, contingent valuation, willingness to pay.

\section{Introducción}

Los helechos forman parte de un grupo de plantas vasculares que reciben el nombre de pteridofitas y que se caracterizan porque en su ciclo de vida presentan alternancia de generaciones independientes y de vida libre, una sexual o esporofítica (que produce esporas) y otra sexual o gametofítica (forma gametos) (Perez \& Reyes, 1993). Además, se definen porque no dependen de otros organismos para su fecundación o dispersión, pues la primera depende del agua y la segunda del viento (Rojas, 2017).

Tal y como lo menciona Muñiz, Mendoza \& Peréz-García (2007), desde tiempos medievales cuando prosperó la brujería, se creía que las esporas de los helechos tenían el poder de proteger de los hechizos y encantamientos mágicos, como la leyenda de San Juan Bautista, consistía en quemar "polvo" de los helechos a media noche en la víspera del nacimiento de un niño con el fin de salvaguardarlo.

Son diversos los servicios ambientales que los helechos representan en el ecosistema y a la vez, esto ha permitido al ser humano utilizarlos de formas muy variadas, desde la utilización del carbón mineral (periodo carbonífero), alimento, medicina, tinte, esencia para perfumes, grasa, fibra, condimento, sustrato o medio de cultivo, planta ornamental y sobre todo como indicador biológico (Hernández \& Nelson, 2007).

Los helechos son parte importante del dosel arbóreo y del sotobosque, de vital importancia en aquellos ecosistemas que juegan un papel sobresaliente en el balance hídrico, en las comunidades vegetales son un componente de la estructura, por lo que conocer su diversidad, distribución y dinámica de sus poblaciones es fundamental para entender los procesos de sucesión y restauración (Tejero, Torres, Mickel, Mehltreter \& Krömer, 2017).

Centroamérica junto con México, constituyen una de las regiones en donde más se concentra la diversidad de los organismos vegetales (Rzedowski, 1991). Y aunque en Honduras aún no se ha inventariado de forma total la diversidad, se tiene un aproximado de 651 especies helechos y licófitos según Nelson et al. (1996). A la vez es importante dar a conocer que se han realizado investigaciones recientes en diferentes áreas protegidas en los cuales se han descubierto nuevas especies y nuevos registros para la flora de Honduras.

Honduras cuenta con un Sistema Nacional de Áreas Protegidas (SINAPH), que tiene 91 áreas, con diferentes categorías de manejo, dentro de las cuales se encuentra el Parque Nacional La Tigra (PNLT), Murillo \& Erazo (2013).

La Tigra es el primer parque nacional de Honduras, declarado mediante Decreto Ley número 976 del 14 de Julio de 1980, con los objetivos de conservar el caudal y la calidad del agua, proteger la biodiversidad y brindar oportunidades de educación ambiental (TSC, 2012). Tiene un área total de 240,4 km2 de los cuales $164,5 \mathrm{~km} 2$ corresponden a la zona de amortiguamiento y 75,9 km2 corresponden a la zona núcleo. EI PNLT se encuentra ubicado entre los municipios de Valle de Ángeles, Santa Lucía, Cantarranas y el Distrito Central (Villeda, 2013) y fue utilizado como referencia para realizar esta investigación, debido a que cuenta con dos estudios sobre helechos realizados por Hernández 2004 y 2006, en donde se ha analizado la parte taxonómica, etnobotánica y ecológica.

Considerando los diferentes usos que se le dan a los helechos (alimento, medicina, plantas ornamentales, entre otros), se decidió realizar esta investigación con el objetivo de Valorar Económicamente los helechos del PNLT, mediante el uso del Método de Valoración Contingente (MVC); conocer la disponibilidad a pagar que tiene la población hondureña por un helecho silvestre y a la vez establecer esta investigación como un dato 
de referencia, para aquellas especies de helechos silvestres para los cuales no existe un mercado definido.

\section{Metodología}

El método VC, consiste en simular un mercado mediante una encuesta a los consumidores potenciales; donde se les pregunta por la máxima cantidad de dinero que pagarían por el bien si tuvieran que comprarlo, como hacen con los demás bienes, de esta forma se deduce el valor que para el consumidor medio tiene el bien en cuestión (Riera, 1994).

Se elaboró un cuestionario (de doce preguntas), estructurado en tres partes 1) Descripción del bien que se pretende valorar, 2) Valoración del bien, 3) Información sobre la persona entrevistada. Para la elaboración del cuestionario se utilizó el programa JotForm, que posteriormente fue enviado vía correo electrónico mediante el enlace proporcionado por el programa, lo cual permitió no incurrir en gastos económicos y tiempo en comparación a realizar las entrevistas personalmente, por teléfono o vía correspondencia impresa.

Los cuestionarios fueron enviados a partir del 16 de febrero al 23 de abril de 2015. La información obtenida se analizó utilizando el programa estadístico SPSS Statistics 20.0, para hacer los análisis de variables cuantitativas (como la media, mediana, la moda, frecuencia, análisis de contingencia y significación) y cualitativas (género, ciudad donde vive, motivos de compra, ocupación entre otros).

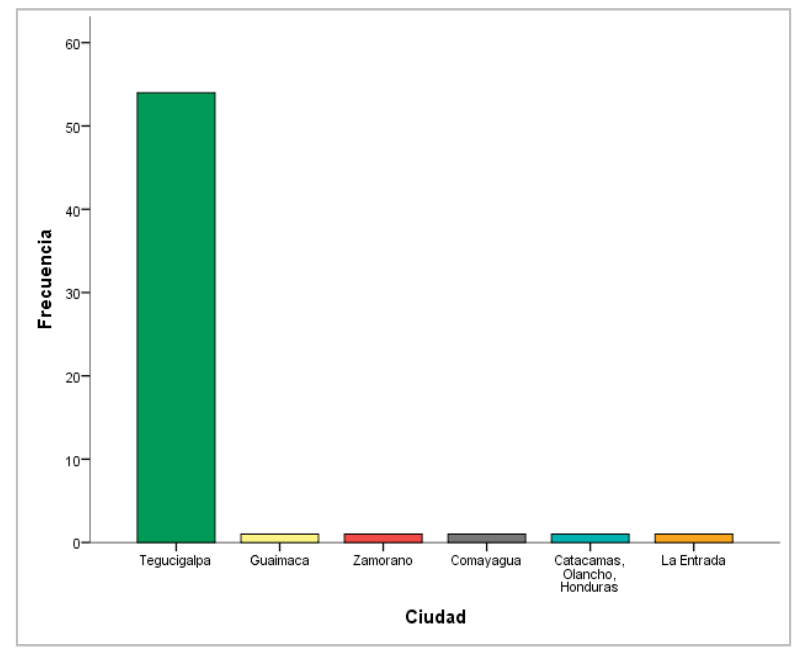

Figura 1. Procedencia de los entrevistados

\section{Resultados}

Se obtuvo un total de 68 entrevistas, que fueron llenadas entre el 04 de febrero y el 21 de abril de 2015, por profesionales universitarios que laboran en diferentes instituciones y/o empresas y estudiantes de la Universidad Nacional Autónoma de Honduras (UNAH). El 53\% de los entrevistados son mujeres y el $47 \%$ son hombres; el $91.5 \%$ vive en la ciudad de Tegucigalpa y el $1.7 \%$ se distribuye en las ciudades de Guaimaca, Zamorano, Comayagua, Catacamas y La Entrada, respectivamente (fig.1), esta característica es importante debido a que el PNLT está ubicado aproximadamente a $12 \mathrm{Km}$ al noreste de la ciudad de Tegucigalpa y cercano a las ciudades de Guaimaca y Zamorano.

La edad promedio de los entrevistados es 28,79 años, la edad media es 24 años y la edad más común es 23 años; la edad mínima es 20 años y la máxima es 76 años (fig. 2).

En respuesta a la pregunta ¿Ha visitado usted el PNLT? El 72,1\% (49) respondió que "Si" y el $27,9 \%$ (19) respondió que "No". Los motivos por los cuales han visitado el PNLT son $51 \%$ recreativo (caminar, disfrutar el paisaje, observar aves y plantas, tomar fotografías, hacer ejercicio), $26,5 \%$ educación y $6,1 \%$ recreación, investigación y buscar productos del bosque y el $4,1 \%$ trabajo y visita familiar (tab. 1), por lo que puede decirse que el PNLT es visitado generalmente por personas jóvenes. Según Maldonado (2000), las actividades recreativas como la observación de plantas y animales, disfrutar del paisaje, tomar fotografias e investigación, son las actividades que mayormente

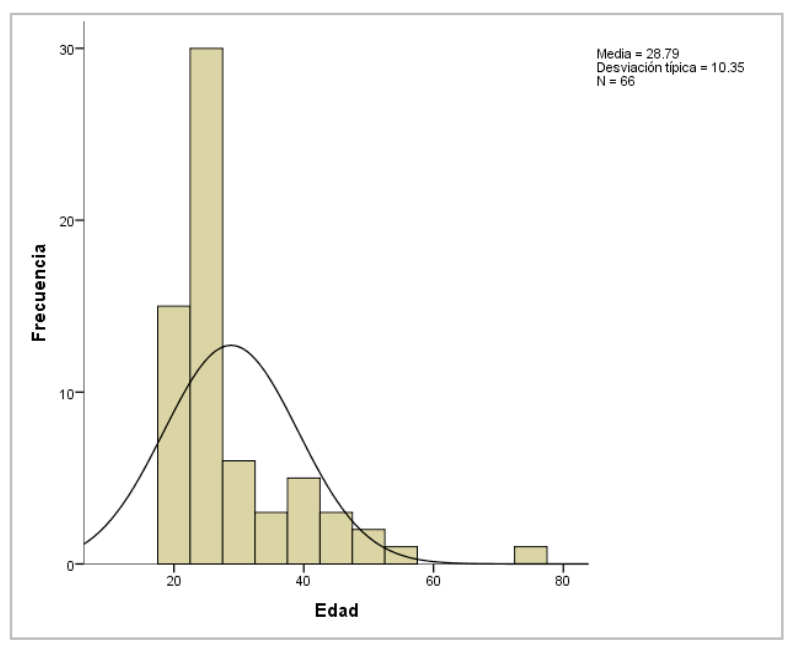

Figura 2. Distribución de la edad de los entrevistados 


\begin{tabular}{|l|c|c|}
\hline Motivos & Fr & \% V \\
\hline Educación & 13 & 26,5 \\
\hline Investigación & 1 & 2 \\
\hline Recreativo & 25 & 51 \\
\hline Recreativo e Investigación & 3 & 6,1 \\
\hline Recreativo y buscar productos del bosque & 3 & 6,1 \\
\hline Trabajo & 2 & 4,1 \\
\hline Visita familiar & 2 & 4,1 \\
\hline Total & 49 & 100 \\
\hline Perdidos & 19 & \\
\hline Total & 68 & \\
\hline Fr $=$ Frecuencia $\% \mathbf{V}=$ Porcentaje Válido & &
\end{tabular}

$\mathbf{F r}=$ Frecuencia, $\% \mathbf{V}=$ Porcentaje Válido

Tabla 1. Motivos de visita al PNLT.

\begin{tabular}{|c|c|c|c|c|}
\hline \multirow{2}{*}{\multicolumn{2}{|c|}{$\begin{array}{l}\text { ¿Compraría } \\
\text { usted } \\
\text { helecho? }\end{array}$}} & \multicolumn{2}{|c|}{$\begin{array}{l}\text { ¿Conoce usted } \\
\text { los helechos? }\end{array}$} & \multirow{3}{*}{$\begin{array}{l}\text { Total } \\
\\
48\end{array}$} \\
\hline & & \multirow{2}{*}{$\begin{array}{l}\mathbf{S i} \\
44\end{array}$} & \multirow{2}{*}{$\begin{array}{l}\text { No } \\
4\end{array}$} & \\
\hline \multirow{2}{*}{$\mathbf{S i}$} & Recuento & & & \\
\hline & $\%$ del total & $65,7 \%$ & $6,0 \%$ & $71,6 \%$ \\
\hline \multirow{2}{*}{ No } & Recuento & 17 & 2 & 19 \\
\hline & $\%$ del total & $25,4 \%$ & $3,0 \%$ & $28,4 \%$ \\
\hline \multicolumn{2}{|c|}{ Total } & $91,0 \%$ & $9,0 \%$ & $100,0 \%$ \\
\hline
\end{tabular}

Tabla 2. Relación entre conocer y comprar un helecho

\begin{tabular}{|c|c|c|c|c|c|}
\hline \multirow{2}{*}{$\begin{array}{ll}\text { ¿Cuánto } & \\
\text { pagaría por un } \\
\text { helecho del } \\
\text { medio silvestre? }\end{array}$} & \multicolumn{4}{|c|}{ Trabaja usted en } & \multirow[b]{2}{*}{ Total } \\
\hline & EP & IG & I & $\mathbf{E}$ & \\
\hline $\begin{array}{ll}\text { L.200,00 } & \mathrm{a} \\
\text { L. } .350 .00\end{array}$ & $5,4 \%$ & $\begin{array}{l}10,8 \\
\%\end{array}$ & $2,7 \%$ & $73,0 \%$ & $91,9 \%$ \\
\hline $\begin{array}{l}\text { L.600,00 } \\
\text { L.1000,00 }\end{array}$ & $0,0 \%$ & $0,0 \%$ & $0,0 \%$ & $2,7 \%$ & $2,7 \%$ \\
\hline $\begin{array}{ll}\text { más } & \mathrm{de} \\
\mathrm{L} .1000,00 & \end{array}$ & $0,0 \%$ & $2,7 \%$ & $0,0 \%$ & $2,7 \%$ & $5,4 \%$ \\
\hline Total & $5,4 \%$ & $\begin{array}{l}13,5 \\
\%\end{array}$ & $2,7 \%$ & $78,4 \%$ & $\begin{array}{l}100,0 \\
\%\end{array}$ \\
\hline
\end{tabular}

$\mathbf{E P}=$ Empresa privada, $\mathbf{I G}=$ Institución del Gobierno, $\mathbf{I}=$ Independiente, $\mathbf{E}=$ Estudiante

Tabla 3. Relación precio de compra y ocupación. se realizan en el PNLT por los visitantes nacionales y extranjeros.

Se elaboró un cuadro de contingencia para determinar si existe relación entre la pregunta ¿Conoce usted los helechos? y ¿Compraría usted un helecho? Se obtuvo un total 67 casos válidos y 1 caso perdido. Se observó que el $91 \%$ de los entrevistados respondió "Si" y de este total el $65,7 \%$ compraría un helecho del medio silvestre; mientras que el 9\% respondió "No" y el 3\% no compraría un helecho del medio silvestre. Para esta relación se obtuvo un valor de $X^{2}=0,777$, lo que indica que existe poca significancia entre las variables (tab. 2).

De las 68 entrevistas realizadas, solo 39 otorgaban un precio a los helechos, según Riera (1994) "No se considerara aquellas respuestas que no otorgan un precio a la situación hipotética planteada"; por lo tanto, únicamente se consideró un total de 39 entrevistas para el análisis económico.

Haciendo una relación entre el género y la pregunta ¿Compraría usted un helecho? y ¿Porque? Se determinó que el $57,1 \%$ de las mujeres compraría un helecho y de este total el $46,4 \%$ lo compraría por motivos ornamentales; el $42,9 \%$ son hombres y de este total el $17.9 \%$ compraría un helecho por motivos ornamentales, 7,1\% para medicina, 3,6\% investigación y el $14,3 \%$ por su belleza (fig. 3). También se pudo determinar que la relación entre las variables es poco significativa debido a que se tuvo un valor $X^{2}=0,100$. En base a lo anterior, se puede decir que los hombres tienen un mayor conocimiento sobre los diferentes usos que se le puede dar a los helechos.

La edad mínima de los entrevistados dispuesto a pagar por un helecho del medio silvestres es de 20 años, la edad promedio es de 25,68 años, la edad media es 23 años y la máxima es de 51 años. La edad más frecuente es 22 años (fig. 4). Este resultado puede ser debido a que existe un mayor número de entrevistas llenada por personas jóvenes.

Se hizo una relación entre la pregunta ¿Cuánto pagaría por un helecho del medio silvestre? y edad de los entrevistados, se obtuvo un total de 37 $(94,9 \%)$ casos válidos, el $91,9 \%$ está dispuesto a pagar (DAP) entre L. 200,00 (8,25 €) a L. 350,00 $(14,45 €)$ y de este total el $21,6 \%$ tiene 22 años, $16,2 \%$ tiene 23 y 24 años de edad respectivamente; el $2,7 \%$ de las personas entrevistadas está dispuesta a pagar entre L. $600,00(24,76 €)$ a L. $1000,00(41,27 €)$ y tienen una edad de 26 años y el $5,4 \%$ de las personas que están dispuestas a pagar más de L. $1.000,00$ y el 2,7\% tiene 45 años de edad (fig. 5). Para esta relación se obtuvo un 


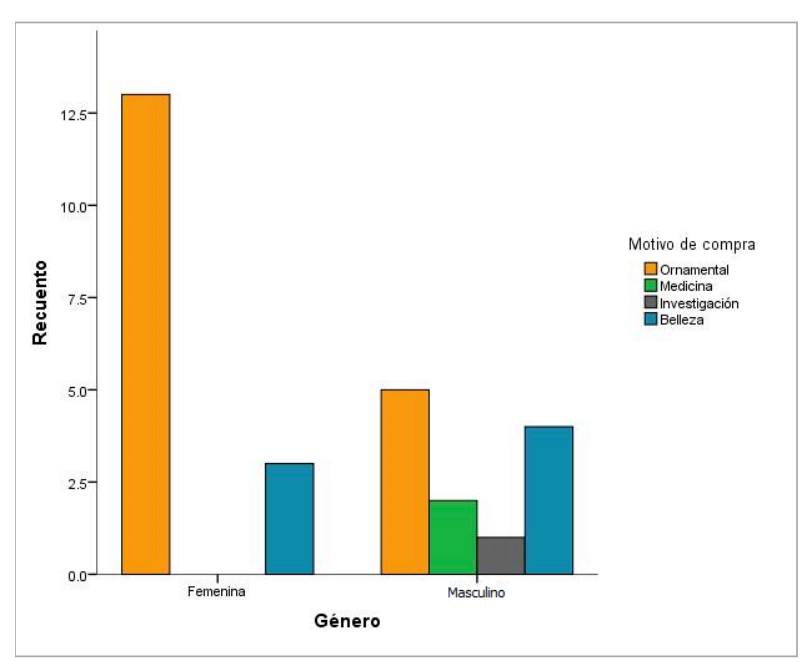

Figura 3. Motivo de compra de un helecho.

valor de $X^{2}=0,027$, por lo que se puede decir que existe una relación significativa entre las variables.

Mediante el análisis del cuadro de contingencia se analizó la relación entre la pregunta ¿Cuánto pagaría por un helecho del medio silvestre? y la ocupación de la persona entrevistada y se observó que el $78,4 \%$ son estudiantes y de este el $73 \%$ pagaría entre L. 200,00 y L.350,00 y el $10,8 \%$ de los que trabajan en una institución del gobierno pagaría la misma cantidad (tab. 3). El valor $\mathrm{X}^{2}=0,843$ por lo que se puede decir que la relación entre las variables es poco significativa estadísticamente. En esta relación hubiese esperado que las personas que trabajan hubieran tenido mayor DAP que los estudiantes, esto puede ser debido a que los estudiantes tienen mayor conocimiento de los helechos, porque el 25,6\% de los entrevistados ha visitado el PNLT por motivos educativos. Es importante destacar que son los estudiantes (universidad y colegio) los que mayormente visitan el PNLT (Maldonado, 2000).

En respuesta a la pregunta ¿Cuánto es lo máximo que estaría dispuesto a pagar? se realizó un análisis de frecuencia del pago máximo que los entrevistados (39) están DAP y se obtuvo un pago promedio de L. 479,20, un valor medio de L. 400,00 que a la vez es el valor más común, que la mitad de los entrevistados esta DAP, el pago mínimo es de L. 200,00 y el máximo es L. 1.500,00. También se observó que el pago máximo tiene una desviación típica de L. 273,20 con respecto a la media y la distribución de los datos es normal positiva (fig. 5).

Haciendo una relación entre la pregunta ¿Cuánto es lo máximo que estaría dispuesto a pagar? $\mathrm{Y}$ el género de la persona entrevistada,

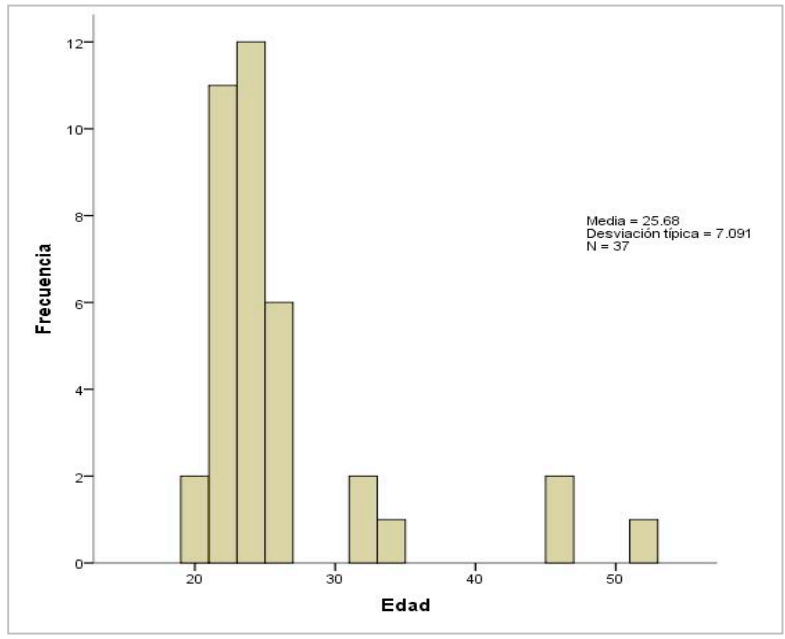

Figura 4. Rango de edad de los entrevistados

se obtuvo un total de $37(94,9 \%)$ casos válidos y $2(5 \%)$ casos perdidos; el 59,9\% son mujeres del cual el $13,5 \%$ pagaría L. 350,00 y el $16,2 \%$ pagaría L.400,00; el 40,5\% son hombres y el 10,8\% pagaría L.400,00 (fig. 6). Para esta relación se obtuvo un valor $\mathrm{X}^{2}=0,545$ indicando así que la relación entre las variables es poco significativa. En base a lo anterior, puede decirse que son las mujeres las que tienen mayor DAP a pesar a que se orientan más hacia el uso ornamental de los helechos.

Un ecosistema puede proveer gran cantidad de servicios, pero su valor económico dependerá finalmente de la disponibilidad y capacidad de pago por parte de los demandantes de tal servicio (Figueroa et al., 2009).

En respuesta a la pregunta ¿Cuál es la máxima cantidad que está dispuesto a aportar para un proyecto de reproducción de helechos silvestres? De los 68 entrevistados se obtuvo un total de 59 casos válidos, 9 casos perdidos. El 44,1\% está dispuesto a aportar más de L. 60,00 y 35,6\% aportaría entre L. 20,00 a L. 40,00 (1,65€), (tab. 4). En base a lo anterior, puede decirse que existe una muy buena percepción hacia los helechos por parte de los entrevistados, porque el $44,1 \%$ esta DAP para apoyar un proyecto de reproducción de helechos silvestres.

Para responder la pregunta ¿Sabía usted que algunos helechos están en peligro de extinción? se estableció una relación entre esta y el género, se obtuvo un total de $65(95,6 \%)$ casos válidos y 3 $(4,4 \%)$ casos perdidos; el $23,1 \%$ de las mujeres y el $24,6 \%$ de los hombres respondieron que "Si" y el $52,3 \%$ de ambos géneros, respondieron que "No" sabían que algunos helechos estaban en peligro 


\begin{tabular}{|l|l|c|c|}
\hline \multicolumn{2}{|l|}{ Aporte } & $\mathbf{F r}$ & $\mathbf{\%} \mathbf{~ V}$ \\
\hline Válidos & L. 5,00 a L. 10,00 & 12 & 20,3 \\
\cline { 2 - 4 } & L.20,00 a L. 40,00 & 21 & 35,6 \\
\cline { 2 - 4 } & más de L. 60,00 & 26 & 44,1 \\
\cline { 2 - 4 } & Total & 59 & 100,0 \\
\hline Perdidos & Sistema & 9 & \\
\hline Total & 68 & \\
\hline
\end{tabular}

Tabla 4. Aporte para un proyecto de reproducción de helechos

\begin{tabular}{|c|c|c|c|}
\hline Motivos & & $\overline{F r}$ & $\% \mathrm{~V}$ \\
\hline & $\begin{array}{lll}\begin{array}{l}\text { Evitar } \\
\text { educación }\end{array} & \text { extinción } & \text { y } \\
\text { edis }\end{array}$ & 14 & 26,9 \\
\hline & $\begin{array}{l}\text { Generar empleo } \quad \mathrm{y} \\
\text { aprovechamiento sostenible }\end{array}$ & 3 & 5,8 \\
\hline & $\begin{array}{lll}\begin{array}{l}\text { Generar empleo y evitar } \\
\text { extracción ilícita }\end{array} & \\
\end{array}$ & 1 & 1,9 \\
\hline & Medicina & 4 & 7,7 \\
\hline & $\begin{array}{llll}\begin{array}{l}\text { Mitigar } \\
\text { silvestre }\end{array} & \text { daño al medio } \\
\end{array}$ & 1 & 1,9 \\
\hline & Muchos beneficios & 1 & 1,9 \\
\hline & Parte del ecosistema & 6 & 11,5 \\
\hline & Potencial económico & 1 & 1,9 \\
\hline Válidos & Producción de Oxigeno & 2 & 3,8 \\
\hline & $\begin{array}{l}\text { Protección } \\
\text { aprovechamiento sostenible }\end{array}$ & 2 & 3,8 \\
\hline & Reproducción & 3 & 5,8 \\
\hline & Retienen agua & 1 & 1,9 \\
\hline & Tener más Diversidad & 1 & 1,9 \\
\hline & $\begin{array}{l}\text { Conservación } \\
\text { concientización }\end{array}$ & 7 & 13,5 \\
\hline & Cumpliendo Ley & 1 & 1,9 \\
\hline & Belleza & 2 & 3,8 \\
\hline & $\begin{array}{l}\text { Ahora se lo importante que } \\
\text { son }\end{array}$ & 1 & 1,9 \\
\hline & Acceso legal & 1 & 1,9 \\
\hline & Total & 52 & 100,0 \\
\hline Perdidos & Sistema & 16 & \\
\hline Total & & 68 & \\
\hline
\end{tabular}

Tabla 5. Motivos para apoyar un proyecto de reproducción de helechos silvestres.

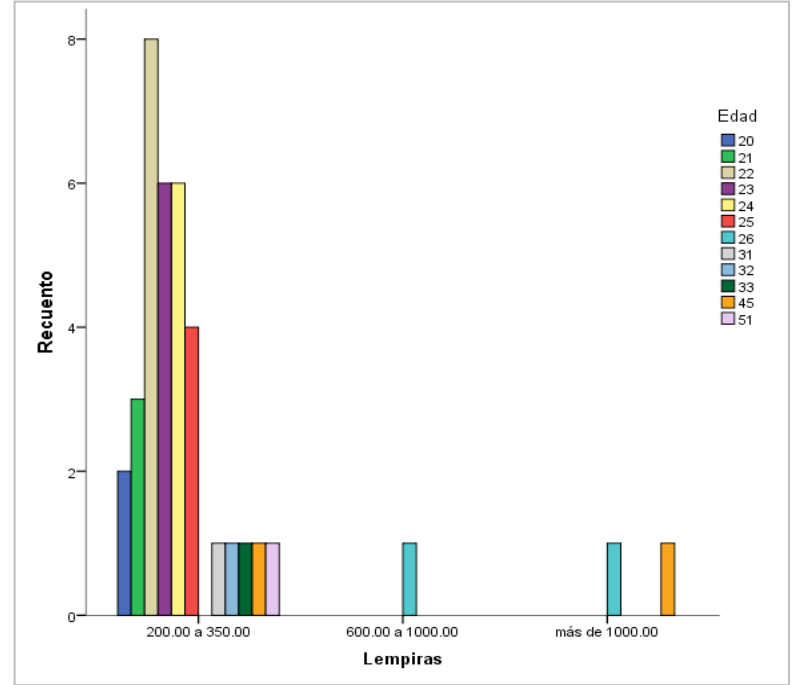

Figura 5. Precio de compra de un helecho vs. edad.

de extinción (fig. 7). Para esta relación se obtuvo un valor de $X^{2}=0,546$. Puede decirse que el $52,3 \%$ de los entrevistados, sin importar el género, no sabían que algunos helechos están en peligro de extinción.

En respuesta a la pregunta ¿Apoyaría usted un proyecto de reproducción de helechos silvestres? y ¿Por qué? Los motivos por los cuales los entrevistados apoyarían el proyecto son un $26,9 \%$ para evitar la extinción y realizar educación, $13,5 \%$ concientización, $11,5 \%$ porque son parte del ecosistema y el 7,7\% para medicina (tab. 5).

Cinco (5) de los 68 entrevistados no apoyaría el proyecto por que manifiestan que: a) No es importante/Necesario (3), b) No por motivos comerciales (1) y c) Se roban el dinero (1), opiniones que fueron consideradas como Respuesta Protesta, esto quiere decir que no están totalmente en desacuerdo con el proyecto. A la vez destacó que las respuesta protestas fueron pocas en relación a otras investigaciones que se han realizado aplicando el MVC.

Estableciendo una relación entre la pregunta ¿Cuánto cree usted que debería pagar como multa la persona natural o jurídica que comercialice ilegalmente con helechos silvestres? y el género, se obtuvo un total de 45 casos válidos $(66,2 \%), 23$ casos perdidos (33,8\%). En donde el $51,1 \%$ de los entrevistados son mujeres y de este total el 13,3\% cree que se debería de pagar una multa entre L.600,00 a L.1.000,00; el 48,9\% son hombres y de este total el $13,3 \%$ cree que se debe pagar más de L.10.000,00 (tab. 6). Para esta relación se obtuvo un valor de $\chi^{2}=0,705$, por lo que se puede decir que la relación entre las variables es poco significativa 


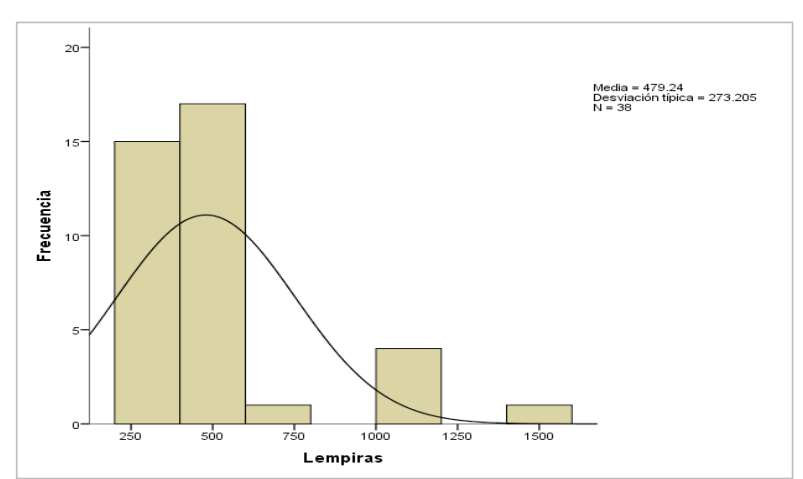

Figura 6. Disponibilidad de un máximo pago.

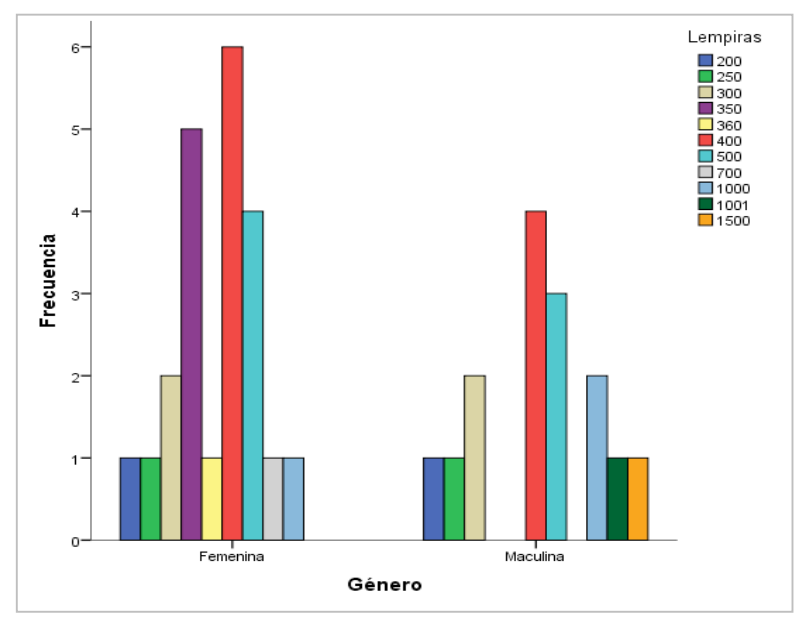

Figura 7. Relación género y máxima DAP.

y a pesar de ello se observa que son los hombres los que asignan la multa más elevada para toda aquella persona natural o jurídica que comercialice ilegalmente con helechos del medio silvestre y esto puede ser debido a que son también ellos los que saben más acerca de los usos que se les puede dar a los helechos (fig. 3). El resultado de esta respuesta puede ser debido al orden de las preguntas, por lo que puede ser considerado un sesgo dentro de la estructura de la entrevista.

La biodiversidad trae a la comunidad nacional e internacional muchos beneficios por el cual muchas personas, empresas y compañías farmacéuticas están dispuesta a pagar por ella (Figueroa et al, 2009).

Tomando como ejemplo los datos obtenidos de la parcela No. 12 del sendero la Esperanza, la cual fue ubicada a 2,120 msnm y en donde se encontró un total de 13 especies, equivalente a un total de 96 individuos (tab. 7), de los cuales 2 especies son arborescentes (63 individuos), 4 especies terrestres (12 individuos), 7 especies son epifitas (21 individuos).

\begin{tabular}{|l|c|c|c|}
\hline \multirow{2}{*}{ Multa } & \multicolumn{2}{|c|}{ Género } & \multirow{2}{*}{ Total } \\
\cline { 2 - 4 } & F & M & \\
\hline L.50,00 a L.500,00 & $8,9 \%$ & $8,9 \%$ & $17,8 \%$ \\
\hline L.600,00 a L.1.000,00 & $13,3 \%$ & $4,4 \%$ & $17,8 \%$ \\
\hline L. $2.000,00$ a L.4.000,00 & $6,7 \%$ & $8,9 \%$ & $15,6 \%$ \\
\hline L. $5.000,00$ a L. $7.000,00$ & $8,9 \%$ & $11,1 \%$ & $20,0 \%$ \\
\hline L.8.000,00 a L. $10.000,00$ & $4,4 \%$ & $2,2 \%$ & $6,7 \%$ \\
\hline más de L. $10.000,00$ & $8,9 \%$ & $13,3 \%$ & $22,2 \%$ \\
\hline Total & $51,1 \%$ & $48,9 \%$ & $100,0 \%$ \\
\hline
\end{tabular}

$\mathbf{F}=$ Femenino, $\mathbf{M}=$ Masculino

Tabla 6. Multa a pagar por comercializar ilegalmente con helechos silvestres

Considerando los datos de la figura 7 en la que el $16,2 \%$ de las mujeres y el $10,8 \%$, de ellas está dispuesta a pagar L. 400,00 por un helecho del medio silvestre (sin hacer la diferencia entre el tipo de hábitat, especie y estado en el que se encuentra el helecho en el ecosistema). En base con lo anterior, se podría obtener un total de L. $38,400.00(1.535,57 €)$.

\section{Conclusiones}

El $91,9 \%$ de los entrevistados estaría DAP entre L. $200,00(8,25 €)$ a L. $350,00(14,45 €)$ y el máximo pago que están dispuestos a realizar es de L, $1.000,00(59,73 €)$. Es importante resaltar que son los estudiantes $(73,0 \%)$ lo que tienen mayor DAP, resultado que desde mi punto de vista es positivo, debido a que pueden ser potenciales compradores de helechos o cualquier otra especie silvestre.

Se puede decir que la mayor parte de los entrevistados (52/68) considera que los helechos son importantes porque son parte del ecosistema que conforma el PNLT (del cual muchos se benefician), por lo que consideran que se debe evitar su extinción y su extracción ilícita, realizar educación y concientización entre otros. Además, el $44,1 \%$ está dispuesto a aportar más de L.60,00 $(2,48 €)$ para apoyar un proyecto de reproducción de helechos silvestres.

Se determinó que la mayor parte de los entrevistados utiliza los helechos como plantas ornamentales, pero el $7,1 \%$ posiblemente lo utilice como plantas medicinales y el 3,6\% para investigación. También se puede decir que el 1,9\% de los entrevistados ve un potencial económico en los helechos, mediante el cual se puede generar empleo a través de un aprovechamiento sostenible, por parte de las personas que viven en 


\begin{tabular}{c|c|l|l|l|c|c|c|c|c|}
\hline No & $\begin{array}{l}\text { P } \\
\text { Subp. }\end{array}$ & \multicolumn{1}{|c|}{ Familia } & Genero & Especie & Cantidad & AB & T & E & Total \\
\hline 1 & 10 & Cyatheaceae & Alsophila & Salvinii & 61 & 61 & & & $\mathbf{6 1}$ \\
\hline 2 & 10 & Cyatheaceae & Cyathea & schiedeana & 2 & 2 & & & $\mathbf{2}$ \\
\hline 3 & 10 & Pteridaceae & Pteris & orizabae & 2 & & 2 & & $\mathbf{2}$ \\
\hline 4 & 10 & Woodsiaceae & Diplazium & drepanolobium & 5 & & 5 & & $\mathbf{5}$ \\
\hline 5 & $10 \mathrm{c}$ & Hymenophyllaceae & Hymenophyllum & fucoides & 1 & & & 1 & $\mathbf{1}$ \\
\hline 6 & $10 \mathrm{c}$ & Dryopteridaceae & Elaphoglossum & peltata & 1 & & & 1 & $\mathbf{1}$ \\
\hline 7 & $10 \mathrm{c}$ & Polypodiaceae & Serpocaulon & loriceum & 1 & & & 1 & $\mathbf{1}$ \\
\hline 8 & $10 \mathrm{c}$ & Dryopteridaceae & Elaphoglossum & eximium & 3 & & & 3 & $\mathbf{3}$ \\
\hline 9 & $10 \mathrm{c}$ & Vittariaceae & Vittaria & graminifolia & 3 & & & 3 & $\mathbf{3}$ \\
\hline 10 & $10 \mathrm{c}$ & Aspleniaceae & Asplenium & harpeodes & 6 & & & 6 & $\mathbf{6}$ \\
\hline 11 & $10 \mathrm{c}$ & Dryopteridaceae & Elaphoglossum & sp. & 8 & & 2 & 6 & $\mathbf{8}$ \\
\hline 12 & $10 \mathrm{c}$ & Dryopteridaceae & Ctenitis & hemsleyana & 3 & & 3 & & $\mathbf{3}$ \\
\hline Total & & & $\mathbf{9 6}$ & $\mathbf{6 3}$ & $\mathbf{1 2}$ & $\mathbf{2 1}$ & $\mathbf{9 6}$ \\
\hline
\end{tabular}

$\mathbf{P}=$ Parcela; Subp= Subparcela; $\mathbf{A B}=$ Arborescente; $\mathbf{T}=$ Terrestre; $\mathbf{E}=$ Epífito. Fuente: Hernández,R. 2004

Tabla 7. Helechos de la parcela No. 12 del sendero La Esperanza del PNLT

las comunidades que se encuentran en la zona de amortiguamiento del PNLT. Además, el 7,7\% opina que los helechos pueden ser utilizados como plantas medicinales.

De acuerdo a la información obtenida durante esta investigación, ya se tiene un precio de referencia para los helechos y específicamente los de la parcela 12 del sendero La Esperanza del PNLT, para el cual se obtuvo un total de L.38,400.00 $(1.535,57 €)$.

Son pocoslosestudios que sehan realizadopara valorar económicamente los recursos naturales y sobre todo un estudio específico para los helechos, por lo que se propone este documento como referencia de aplicación del Método de Valoración Contingente (MVC), y la generación de un valor cuantitativo para los helechos, el cual puede ser utilizado por el Estado de Honduras para solicitar la retribución de benéficos económicos, científicos y otros (en relación al CDB), de algunas empresas farmacéuticas nacionales e internacionales que han utilizado el recurso fitogenético del país, para generar productos con altos márgenes de ganancias. Además, esta metodología puede ser usada para analizar otras especies de interés que están en peligro de extinción, o que son comercializadas a nivel nacional e internacional (formando parte de los Apéndices de CITES), debido a su comercio internacional.

Además, con este documento se dispone de una herramienta más para que se haga una gestión adecuada de estos, por parte de las instituciones encargadas de la administración de los recursos naturales, por parte de la sociedad, los operadores de justicia y los tomadores de decisiones políticas.

\section{Recomendaciones}

Realizar un estudio de mercado para conocer cuáles son las especies de helechos más apetecidas por los pobladores hondureños y las preferencias del mercado internacional, para así desarrollar un proyecto de reproducción de helechos de forma sostenible y poder comercializarlos legalmente.

Consultar con los pobladores de las comunidades circundantes sobre el proyecto de aprovechamiento sostenible de helechos y gestionar fondos para realizarlo.

Realizar investigaciones fisicoquímicas de los helechos, para conocer su potencial económico y a la vez, conocer cuáles son los principios activos que cada uno de estos posee, para que puedan ser utilizados ampliamente como plantas medicinales o alimenticias, como es el caso de Phlebodium pseudoaureum y $P$. decumanum.

\section{Agradecimientos}

Esta investigación se realizó gracias a la beca otorgada por el Programa de becas Iberoamérica+Asia+Universidad de Valladolid+Banco de Santander. Al apoyo brindado por la Dra. Beatriz Urbano López De Meneses y al Dr. Alexander Francisco Rojas Alvarado, por los conocimientos transmitidos. Al MSc. Miguel Antonio Barahona, profesor de la Universidad 
Nacional Autónoma de Honduras (UNAH) y a sus alumnos por ayudarme a llenar las entrevistas.

Al Dr. Alejandro Zavala Hurtado de la Universidad Autónoma Metropolitana de Iztapala, México, por sus recomendaciones. Quiero agradecer especialmente al profesor Salvador Hernández por su calidad humana y los consejos brindados. A los profesores Enrique Relea y Luis Manuel Navas por su apoyo y apertura.

A mi familia por su apoyo incondicional y finalmente doy gracias a todas aquellas personas que de alguna manera se involucraron en la realización de esta investigación.

\section{Referencias}

Figueroa, B., Reyes, P. \& Rojas, J. (2009) Pago por servicios ambientales en áreas protegidas en América Latina. Documento Técnico, FAO, Santiago, Chile.

Hernández, R. (2004) Diversidad de helechos en el sendero La Esperanza del Parque Nacional La Tigra. Tesis de Licenciatura, Universidad Nacional Autónoma de Honduras (UNAH). Tegucigalpa.

Hernández, R. (2006) Diversidad de Helechos en el sendero La Cascada del Parque Nacional La Tigra. USAID/MIRA, Tegucigalpa.

Hernández, R. (2015) Valoración económica de los helechos del parque nacional La Tigra, Honduras, C.A. Tesis. Universidad de Valladolid,

Hernández, R. \& Nelson, C. (2007) Etnobotánica de los helechos de Honduras. Ceiba, 48(1-2), 1-10.

Maldonado, R. (2000) Determinación de la capacidad de carga turistica como una opción para el manejo sustentable del Parque Nacional La Tigra. Tesis de Maestria, CATIE, Turrialba.

Muñiz, M., Mendoza, A. \& Peréz-García, B. (2007) Uso de los helechos y plantas afines. Etnobiología, 5, 117-125.

Murillo, A. \& Erazo, M. (Eds.). (2013) Anuario Estadístico Forestal. Tegucigalpa: Instituto Nacional de Conservación y Desarrollo Forestal, Áreas Protegidas y Vida Silvestre (ICF).

Perez, B. \& Reyes, I. (1993) Helechos: propagación y conservación. Ciencias, 11-17.

Riera, P. (1994) Manual de Valoración Contingente. Madrid: Instituto de Estudios Fiscales.

Rojas, A. (2017) Observatorio ambiental de la Universidad Nacional de Costa Rica. Obtenido de: http://www.observatorioambiental.una.ac.cr/index. php/indicadores-ambientales/tema/357-variacion-enla-diversidad-y-distribucion-de-licofitos-y-helechosen-la-estacion-biologica-rio-macho-y-su-relacion-convariaciones-climaticas

Rzedowski, J. (1991) Diversidad y origenes de la flora fanerogamica de México. Acta Bot. Mexica, 14, 3-21.

Tejero, D., Torres, A., Mickel, J., Mehltreter, K. \& Krömer, T. (2017) Helechos y licopodios. Obtenido de: http:// www.academia.edu/11871666/Helechos_y_licopodios

Tribunal Superior de Cuentas (TSC) -2012- Auditoría de gestión ambiental practicada a el Instituto de Conservación Forestal (ICF) sobre la administración del área protegida Parque Nacional La Tigra (PNLT). Auditoria, TSC, Auditorias al Sector Recursos.

Villeda, E. (2013) Plan de Manejo del Parque Nacional La Tigra. Tegucigalpa: Instituto Nacional de Conservación y Desarrollo Forestal, Áreas Protegidas y Vida Silvestre (ICF). 
